\title{
COVID 19 in Liver Transplant Patient - Case Report
}

\section{Ratiani ${ }^{1,2}$, V Shoshiashvilit,2*, E Vashakidze ${ }^{1,2}$, M Rurua ${ }^{1,2}$, E Pachkoria $^{1,2}$, E khuchua ${ }^{1}$, L Gabunia ${ }^{1,2}$ and K Machavariani ${ }^{1,2}$}

${ }^{1}$ First University Clinic of Tbilisi State Medical University, Georgia

${ }^{2}$ Tbilisi State Medical University, Georgia

*Corresponding Author: V Shoshiashvili, First University Clinic of Tbilisi State

Medical University, Georgia.
Received: July 16, 2021

Published: September 08, 2021

(C) All rights are reserved by $\mathbf{V}$ Shoshiashvili., et al.

\begin{abstract}
Coronavirus disease-2019 (COVID-19) caused by coronavirus 2 (SARS-CoV-2) infection is a global health problem; Severe COVID-19 can lead to multi-organ failure and may be associated with high mortality. The incidence and outcomes of COVID-19 in immunocompromised patients are a matter of debate, clinical presentation and management of liver injury in liver transplant patients with COVID -19 is unclear and may be an increasing challenge for the transplant centers. This is a Case report from Georgia that presents a case of COVID-19 in a patient with a liver transplant. Due to Corticosteroids and Antibiotic therapy, in parallel with continuing immunosuppressive drug full recovery was achieved. His maintenance immunosuppressive therapy includes calcineurin inhibitor (Cyclosporine) 75 mg BID, which was not ceased.
\end{abstract}

Keywords: COVID-19; Liver Transplantation; Immunosuppressants; Management

\section{Introduction}

Coronavirus disease-2019 (COVID-19) caused by coronavirus 2 (SARS-CoV-2) infection is a global health problem [1]; Severe COVID-19 can lead to multi-organ failure and may be associated with high mortality [2]. In Georgia as of July 9 we have confirmed 374.836 cases of COVID 19; recovered 357.804; fatal outcome registered in 5421 cases [3]. Age, severity of the infection and comorbidity are important predictors of poor outcomes in COVID-19 [4]. The incidence and outcomes of coronavirus disease 2019 (COVID-19) in immunocompromised patients are a matter of debate [5]. Liver injury is common in patients with COVID -19 [6], but clinical presentation and management of liver injury in liver transplant patients with COVID 19 is unclear and may be an increasing challenge for the transplant centers [7]. In this report we have described liver transplant case with COVID 19.
Case

A 59-year-old man was admitted to the TSMU First University Clinic (Tbilisi, Georgia) in viral replication stage of the disease (on the fourth day from the onset of the disease) with the history of a fatigue, chills, fever, sweating, muscle and back pain. In addition, in 2009 the patient underwent orthotopic liver transplantation due to decompensated liver cirrhosis. his maintenance immunosuppression therapy includes calcineurin inhibitor (Cyclosporine) $75 \mathrm{mg}$ BID. Co- morbidities: Diabetes type 2; Arterial Hypertension.

His past medical history was notable for a chronic hepatitis $\mathrm{C}$ (which was successfully treated with antiviral treatment and a sustained virological response -SVR was achieved). The patient had no history of any respiratory symptoms, including cough and dyspnea, as well as high blood pressure or coronary artery disease. On admission, the following vital signs were obtained: temperatu- 
re $37.9^{\circ} \mathrm{C}$; pulse, 80 beats per minute; respiratory rate, 16 breaths per minute; blood pressure,115/70 mm Hg; SPO2 - 97\%; The patient was admitted to hospital for farther evaluation, and the nasal and pharyngeal swab samples were collected to test for COVID-19 using real-time polymerase chain reaction (RT-PCR), diagnostic testing resulted positive for COVID-19. Markers of inflammation within normal limits; GGT- 323.2 is moderately elevated (8-61 U/l); HBSAg, HBc (tot) Ab; HIV Ab were negative (Table 1). Computed tomography (CT) scan of the chest revealed bilaterally, focal infiltra- tes of varying sizes, as well as several patchy ground-glass opacities. Lung damage index -4 points. Pursuant to cholestatic pattern of liver function test abdominal Ultrasound was performed. Liver: the oblique-vertical dimension of the right part: $165 \mathrm{~mm}(\mathrm{~N}<150$ $\mathrm{mm} \mid$ ), cranio-caudal dimension of the left part: $90 \mathrm{~mm}(\mathrm{~N}<100 \mathrm{~mm})$, angles rounded. The echoic structure of the liver parenchyma is homogenous. Echogenicity increased. Suture damage: not observed. Vascular picture of the liver: normal. The diameter of Vena Porta: $20 \mathrm{~mm}(\mathrm{~N}<14 \mathrm{~mm})$, intrahepatic and extrahepatic bile ducts - not dilated; Spleen-140X $50 \mathrm{~mm}$.

\begin{tabular}{|c|c|c|c|c|c|c|c|c|}
\hline \multicolumn{4}{|c|}{ Virus replication stage } & \multicolumn{5}{|c|}{ Immune Phase } \\
\hline & 10.03 .21 & 12.03 .21 & 15.03 .21 & & 20.03 .21 & 23.03 .21 & 26.03 .21 & 29.03 .21 \\
\hline WBC & 4.45 & & 5.68 & 3.9 & 4.51 & 7.75 & 6.98 & 6.67 \\
\hline LYMPH\% & 40.8 & & 28.6 & 21.7 & 31.8 & 26.1 & 28.5 & 24.4 \\
\hline NEU \% & 49.3 & & 68.8 & 73.3 & 58.1 & 63.7 & 69 & 66.8 \\
\hline HGB g/dl & 10.7 & & 10.8 & 10.1 & 10.5 & 10.9 & 9.6 & 10.3 \\
\hline HCT l/L & 33.3 & & 33.5 & 30.9 & 31.6 & 32.8 & 29.3 & 31 \\
\hline PLT & 150 & & 125 & 144 & 182 & 283 & 257 & 246 \\
\hline CREA nmol/L & 155 & 147.1 & 148.5 & 126.3 & 106 & 152.8 & 129.9 & 137.8 \\
\hline AST u/L & 28.2 & & 21.8 & 30 & 18.9 & 22.1 & 14.6 & 45.6 \\
\hline ALT u/l & 21.5 & & 18.7 & 34.2 & 24.4 & 20.5 & 14.8 & 47.6 \\
\hline$y-G G T u / L$ & 323.2 & & 338.1 & 446 & 494.2 & 582.1 & 446.6 & 504.6 \\
\hline T.BIL & 15.4 & & 13.9 & 15.8 & & 16.1 & 17 & 18.1 \\
\hline $\mathrm{CRP} \mathrm{mg} / \mathrm{L}$ & 7 & 8 & $<5$ & 38 & & 116 & 15 & 12 \\
\hline LDH u/L & 109 & & & & & 232 & & 191.9 \\
\hline D-Dimer ng/ml & 335 & & 195 & 383 & 697 & 1830 & 756 & 515 \\
\hline APTT s & 40.3 & 44.6 & 46.3 & 41.8 & 41.9 & 48.8 & 41.4 & 49.7 \\
\hline INR & 1.02 & 1.16 & 1.04 & 1 & 1 & 1.22 & 1.02 & 1.16 \\
\hline FB g/l & 2.5 & 3.5 & 2.6 & 5.1 & 4.8 & 5.3 & 4.9 & 5.4 \\
\hline PCT ng/ml & & $0.5-2.0$ & $<0.5$ & $<0.5$ & $<0.5$ & $05-2.0$ & $<0.5$ & $<0.5$ \\
\hline UREA mmol/L & & & 9.75 & 7.85 & 7.85 & 9.65 & 9.87 & 10.96 \\
\hline Ferritin ng/ml & & & 413.3 & 450.8 & & 706.3 & & 726 \\
\hline TP g/l & & & 76.2 & & & & & \\
\hline ALB g/l & & & 44.38 & & & & & \\
\hline Cortisol ng/dl & & & & & & 13.67 & & \\
\hline
\end{tabular}

Table 1: Laboratory findings.

The patient was transferred to an isolation room. Supportive therapy was initiated. Calcineurin inhibitor 75 mg BID was not sus- pended. on the third day of the disease Procalcitonin (PCT) was 0.5-2; Empirically, antibiotic therapy with cefoperazine-sulbactam $2 \mathrm{~g}$ 
IV BID was started. on the fifth day PCT was in normal range $<0.5$ monitoring and correction of glucose level, electrolytes and ABG was carried on. Patient's condition improved. The temperature dropped up to $36.6 \mathrm{C}$; He had no cough, or any other symptoms.

Treatment was continued with antibiotic, dexamethasone $8 \mathrm{mg}$ daily, anticoagulant Nadroparine calcium (Fraxiparine) $-0.4 \mathrm{mg}$ once a day.

According to lab. findings CRP was mildly elevated, GGT - 494.2; ALT, AST and other findings were in normal range on 23.03.2021 (16 ${ }^{\text {th }}$ day of disease) -High fever (38.5C), fatigue and nonproductive cough revealed. The patient's vital signs were as follows: temperature, $38^{\circ} \mathrm{C}$; heart rate, 88 beats per minute, respiration rate, 22 beats per minute; blood pressure, $120 / 70 \mathrm{~mm} \mathrm{Hg}$; SPO2 - $90 \%$ without oxygen support.

According to lab tests markers of inflammation were elevated (PCT 0.5-2; CRP - 116 mg/l; D dimer- 1830 ng/ml; Ferritin- 706.3 ug/l;).

Serological tests for EBV, HSV1 and HSV2 was performed- there was no active infection Conforming to the chest CT scan: The heart size is not increased, the amount of fluid in the pericardium is within the norm. There is no mediastinal lymphadenopathy. Bilaterally, focal infiltrative changes of various small, different localization, ground glass opacities are revealed in all lung fields. Free fluid and air are not observed in the pleural cavity, lung damage index - 12 points.

According to the magnetic resonance imaging of the abdomen, liver cranio-caudal dimention $-16 \mathrm{~cm}$, maximum axial dimension - 14.5/20.8cm. Intrahepatic bile ducts are not dilated. The portal vein is dilated on axial dimension in $3.2 \mathrm{~cm}$. Spleen enlarged, cranio caudal size is $17 \mathrm{~cm}$. mild fluid effusion was seen in the Abdomen.

Thus, CT scan identified progression of pneumonia from 4 to 12 points; Markers ofinflammation were significantly elevated.

In spite of Interleukin 6 was norm, due to increased CRP, D dimer and Ferritin we can consider the Picture of Cytokine Storm. PCT - 0.5-2 - Methylprednisolone $500 \mathrm{mg}$ was administered intravenously once Meropenem - 1g IV q 8hr was administered empirically; dexamethasone $8 \mathrm{mg}$ per day; Oxygen therapy (3-4 l/per minute), anticoagulant Nadroparine calcium(Fraxiparine-0.4mg BID) correction of the electrolyte imbalance was initiated; calcineurin inhibitor (Cyclosporine) 75 mg BID was not ceased.

In 3 days -Temperature: $37.1 \mathrm{C}$, HR- 77; T/A- 117/70 mm/hg; Oxygen Saturation SPO2 - 97\% (without oxygen supply). Additionally, improved the results of the Lab tests - PCT<0.5; CRP- $12 \mathrm{mg} / \mathrm{l}$; D dimer-515 ng/ml; (Table 1) Abdominal Ultrasound showed no evidence of Ascites. According to the chest CT scan the volume and degree of diffuse infiltrative changes of various sizes in the lung are reduced bilaterally, and the existing dense consolidation sites are also displaced. There is no free fluid and air in the pleural cavity bilaterally. Lung damage index - 10 points. Patient recovered and on 29.03.2021 (on the $19^{\text {th }}$ day of the disease) was discharged to home.

\section{Discussion}

Liver Transplantation (LT) remains the only curative treatment for selected patients with end-stage liver diseases and acute liver failure. Survival rates have improved significantly in the last 25 years. Advances in surgical techniques, the introduction of new immunosuppressive agents, and the early diagnosis and management of complications after LT are the main factors contributing to such great success [8]. Theoretically, liver transplanted patients may have a more significant viral burden and prolonged shedding of the virus resulting in higher infectivity. The impact of immunosuppression in the post-transplant setting is currently unknown; however, there is a concern that immunocompromised patients are at a higher risk of morbidity and mortality due to COVID-19 infection, although data on COVID-19 in liver transplant patients are scarce [9]. A case series, from Italy, showed that children who had received liver transplants, despite being immunosuppressed, were not at increased risk of severe pulmonary disease than the general population suggesting that immunosuppression may protect against cytokine storm induced by COVID-19, which is responsible for the severe illness [10]. Moreover, three of 111 long-term liver transplant survivors (transplanted more than 10 years ago) have died following severe COVID-19 disease. All three were male, older than 65 years, receiving antihypertensive drugs, overweight (BMI $>28 \mathrm{~kg} / \mathrm{m} 2$ ), with hyperlipidemia and diabetes (median HbA1c of $6.9 \%$ ), and their immunosuppressive regimen had been gradually tapered off, with deficient concentrations of calcineurin inhibitors [11]. Most require hospital admission and approximately 15\% intensive care unit admission; mortality has been observed only in patients 60 years of age or older and was higher among males. By 
contrast, three of 40 recently transplanted (i.e., within the past 2 years) patients have tested COVID-19-positive, and although quarantined, all are experiencing an uneventful course of disease [12]. It was noticed that, despite concerns that liver transplant (LT) recipients may be at high risk of unfavorable outcomes from COVID-19 due to the high prevalence of co-morbidities, immunosuppression, and aging, a detailed analysis of their effects in large studies is lacking. More epidemiologic data of COVID-19 in transplant recipients will be forthcoming. It is not clear why, in mentioned reports, transplanted patients with COVID-19 have fewer complications than expected. According to the Spanish study, upon hospital admission, mycophenolate dose reduction or withdrawal could help in preventing of severe COVID-deleterious effect but it was not observed with calcineurin inhibitors or everolimus and complete immunosuppression withdrawal showed no benefit [13].

Our patient, 59 -year old male, with a liver transplant, with Co-morbidities: Diabetes type 2; Arterial Hypertension. Due to Corticosteroids and Antibiotic therapy, in parallel with continuing immunosuppressive drug full recovery achieved. His maintenance immunosuppressive therapy includes calcineurin inhibitor (Cyclosporine) $75 \mathrm{mg}$ BID, which was not ceased; liver function was not significantly deteriorated. Despite all above mentioned risk factors the outcome was good, the patient recovered.

\section{Conclusion}

In conclusion, Mortality rates were lower than those observed in the age- and gender-matched general population, thereby suggesting that chronic immunosuppression could exert a certain protective effect against the most severe forms of COVID-19, but further research is needed to prove this hypothesis [14]. Being chronically immunosuppressed and with increased comorbidities, LT patients are more likely to develop COVID-19 within an outbreak scenario and therefore social distancing and other preventative measures should be enhanced in these patients.

\section{Bibliography}

1. https://www.who.int/emergencies/diseases/novel-coronavirus-2019

2. WHO. Clinical Management of COVID-19: Interim Guidance, 27 May 2020. World Health Organization (2020).
3. https://stopcov.ge/

4. de Bruin S., et al. "Clinical features and prognostic factors in Covid-19: A prospective cohort study". Lancet 67 (2021): 103378.

5. D’Antiga L. "Coronaviruses and immunosuppressed patients. The facts during the third epidemic". Liver Transplantation 26 (2020): 832-834.

6. Zhang C., et al. "Liver injury in COVID-19: management and challenges". Lancet Gastroenterology and Hepatology 5 (2020): 428.

7. Adam R., et al. "Evolution of indications and results of liver transplantation in Europe. A report from the European". (2012): 688.

8. Van Doremalen N., et al. "Aerosol and surface stability of SARSCOV-2 as compared with SARS-COV- 1". The New England Journal of Medicine 382 (2020): 1564-1567.

9. D’Antiga L. "Coronaviruses and immunosuppressed patients. The facts during the third epidemic". Liver Transplantation (2020).

10. Bhoori S., et al. "COVID-19 in long-term liver transplant patients: preliminary experience from an Italian transplant centre in Lombardy". Lancet Gastroenterology and Hepatology 5.6 (2020): 532-533.

11. Liu B., et al. "Successful treatment of severe COVID- 19 pneumonia in a liver transplant recipient". American Journal of Transplantation 20.7 (2020): 1891-1895.

12. Jordi Colmenero., et al. "Epidemiological pattern, incidence, and outcomes of COVID-19 in liver transplant patients". Journal of Hepatology 74.1 (2021): 148-155.

13. Mehta P., et al. "COVID-19: consider cytokine storm syndromes and immunosuppression". Lancet 395.10229 (2020): 1033-1034.

14. https://www.jhsph.edu/news/news-releases/2021/covid19-outcomes-for-patients-on- immunosuppressive-drugs-onpar-with-non-immunosuppressed-patients.html

Volume 5 Issue 10 October 2021 (C) All rights are reserved by $\mathrm{V}$ Shoshiashvili., et al. 